\title{
Pre-swirl mechanism in front of a centrifugal compressor: effects on surge line and on unsteady phenomena in surge area
}

\author{
Amélie Danlos ${ }^{1, *}$, Pierre Podevin ${ }^{1}$, and Michel Toussaint ${ }^{2}$ \\ ${ }^{1}$ Conservatoire National des Arts et Métiers, Laboratoire de Chimie Moléculaire, Génie des Procédés Chimiques et Energétique \\ (CMGPCE - EA7341), 292 Rue Saint Martin, 75003 Paris, France \\ ${ }^{2}$ Association Technique Turbine à Gaz (ATTAG), 15 rue Marat, 78210 Saint-Cyr-1’Ecole, France
}

\begin{abstract}
Using a pre-swirl mechanism upstream an impeller of a compressor allows to modify its characteristics curve, while weakly damaging its efficiency. Another consequence of the pre-swirl is to push back the surge line limit and to increase the operation zone towards the low flow rate limits. A centrifugal compressor has been modified in order to add a swirl generator device upstream the impeller. The incidence values of blades can vary from $0^{\circ}$ (no pre-swirl) to $\pm 90^{\circ}$. The variation of the stator blades incidence has several main consequences: to allow a flow rate adjustment with a good efficiency conservation, to increase the angular velocity with a constant shaft power, to produce a displacement of the surge line limit. In this paper, the results of experimental studies are presented to analyze the surge line and the intensity of unsteady phenomena when the compressor works in its surge area.
\end{abstract}

\section{Nomenclature}

$\pi \quad$ compression ratio;

$q_{m} \quad$ mass flow rate;

$N \quad$ rotation speed;

$R P M$ rotation per minute;

$\omega \quad$ angular velocity;

$\delta \quad$ pre-swirl angle;

$P \quad$ shaft power;

$U_{1}, U_{2}$ respectively entrainment velocity at the inlet and the outlet of the compressor wheel;

$V_{1}, V_{2}$ respectively absolute velocity at the inlet and the outlet of the compressor wheel;

$\alpha_{1} \quad$ angle between $U_{i}$ and $V_{i}$ at the compressor wheel inlet;

$\alpha_{2} \quad$ angle between $U_{i}$ and $V_{i}$ at the compressor wheel outlet;

$\beta_{1} \quad$ angle between $U_{i}$ and $W_{i}$ at the compressor wheel inlet.

\section{Introduction}

In order to reduce pollutant emissions and to decrease fuel consumption, a large part of the current automotive market is equipped with supercharged engines. Turbochargers used for these efficient systems have numerous advantages but their working range has two limits imposed by choke conditions for very high mass flow rates and surge phenomenon for low mass flow rates. This phenomenon is translated by instabilities in the turbocharger, with the appearance of reversed flow at the inlet. Deep surge is very dangerous for the system because of the mechanical damages caused by these instabilities. It is then necessary to find a way to push back this low limit of working range. Many devices have been studied $[1,2]$. The pre-swirl, also called inlet guide vane, is the solution chosen for this study.

The pre-swirl, using stator blades with variable angle of attack at the upstream of a compressor wheel engine, is a technique mainly applied to improve the flow rate adjustment and the power of large impellers. This method allows to obtain an angular deviation of the absolute velocity of the fluid upstream the leading edge of the compressor wheel blades (Fig. 1).

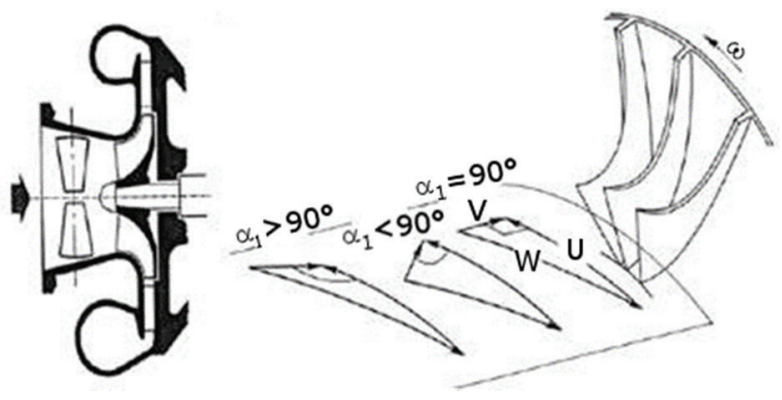

Fig. 1. Theoretical diagram of a pre-swirl system.

The consequence of this angular deviation is to modify, in accordance with Euler's equation, the compressor power input $P$ with our experimental device (Equation 1)

$$
P=q_{m}\left(U_{2} V_{2} \cos \alpha_{2}-U_{1} V_{1} \cos \alpha_{1}\right) .
$$

Without pre-swirl, as $\alpha_{1}$ is theoretically equal to $90^{\circ}$, the second term of Euler's equation is zero (at least at

*Corresponding author: amelie.danlos@lecnam.net 
nominal point). If angle $\alpha_{1}$ is no longer equal to $90^{\circ}$ but takes the value imposed by the pre-swirl blades, it is immediately checked that the compressor power input increases or decreases according to the direction (angle $\delta$ ) chosen by the user. In this paper, the positive pre-swirl represents values of $\delta$ which deflect the flow in the direction of angle $\beta_{1}$ of the blades at the compressor wheel inlet. Then, negative pre-swirl describes the deflection of the flow in the other direction. Experimental measurements presented in this paper have been carried out for three relatively low rotation speeds, in order to prevent compressor damage. Indeed, during measurements, when the compressor works in its surge area it is dangerous for the compressor itself but also for its associated circuit $[3,4]$. Thus, in this paper, we focus on compression ratio curves in order to visualize and analyze the surge line limit.

\section{Compressor surge phenomenon}

All compressors forced to operate at very low flow rate are submitted to a very unsteady and dangerous regime $[3,4]$. This aerodynamic instability has as its consequence, among others, strong fluctuations of pressure and flow rate. The inlet geometry of the circuit upstream the turbocharger has effects on the appearance of surge phenomenon [5]. It is then important to optimize this inlet.

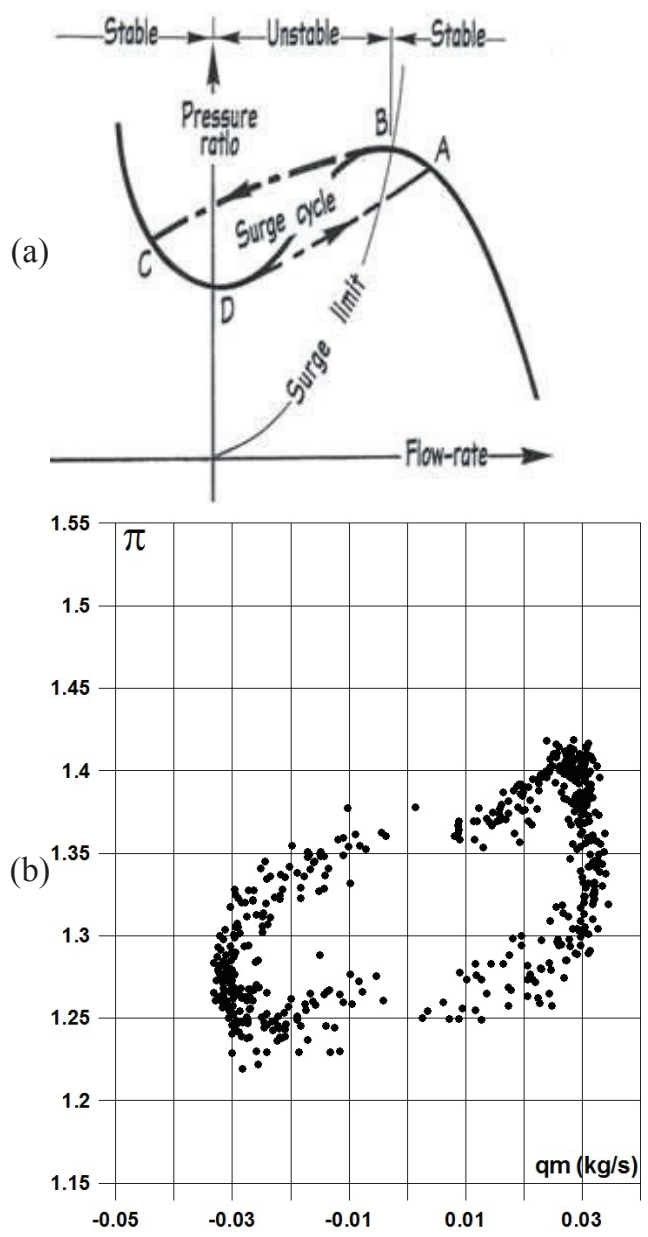

Fig. 2. Description of the surge loop: (a) Theoretical characteristic curve, (b) characteristic curve.
The unstable operating point characterizing deep surge describes a loop (Fig. 2) with an amplitude and a frequency dependent on the associated circuit [6-8].

\section{Experimental device}

The measurements have been conducted on a test bench composed of a pre-swirl located near the turbocharger inlet and a flow control valve placed downstream the turbocharger in order to control the flow rate introduced in the system. This device allows to modify the flow rate to use the turbocharger in its working range or even in the deep surge. Many sensors are used to measure different values at each component of the circuit: pressure sensors at the inlet and outlet of each component, temperature sensors and a flowmeter (placed upstream the pre-swirl). The pre-swirl device used has nine blades (Fig. 3). All blades are controlled by the same screw guidance to rotate all blades with the same angle to their own axes. The incidence angle of each blade can then be imposed by the user for different measurements. These blades can rotate at $360^{\circ}$ around their axes.

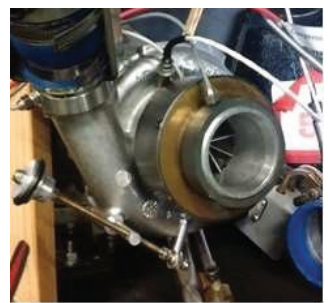

(a)

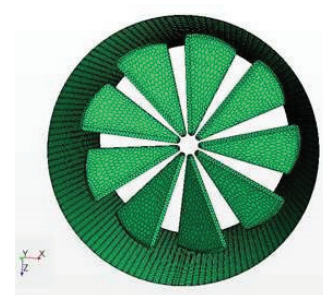

(b)
Fig. 3. Pre-swirl device used at compressor inlet in the test bench: (a) picture of the pre-swirl inlet, (b) pre-swirl diagram in sketch.

\section{Results}

\subsection{Effect of the pre-swirl on the surge line limit}

The experimental results are presented in Fig. 4. These first measurements have been carried out for four arbitrarily chosen values of pre-swirl angle: $26^{\circ}, 52^{\circ},-26^{\circ}$ and $-52^{\circ}$. A short analysis of these results confirms the effect of the pre-swirl of the flow upstream the compressor wheel on the appearance of the surge line limit. A pre-swirl in the flow direction at the compressor wheel inlet allows to push back this limit. This movement of the surge limit is accompanied by the light improvement of the compression ratio for a moderate preswirl angle.

In the case of a negative pre-swirl, the surge line limit is pushed back too for a considerable angle as for example with an angle of $-52^{\circ}$. However, this angle of pre-swirl induces a large decrease of the compressor performances. On the other hand, a moderate negative pre-swirl has beneficial effects on the surge limit since it moves back in this case. The main challenge of this problem is to find the optimal angle to push back the appearance of surge phenomenon but also to improve compressor performances. 
(a)

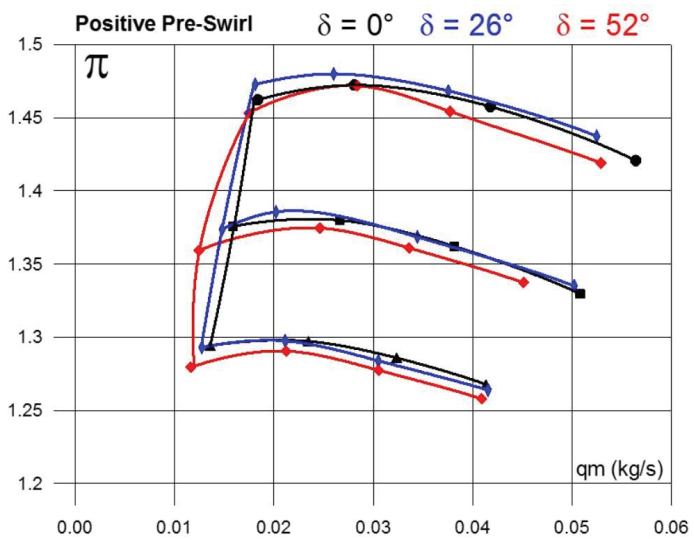

(b)

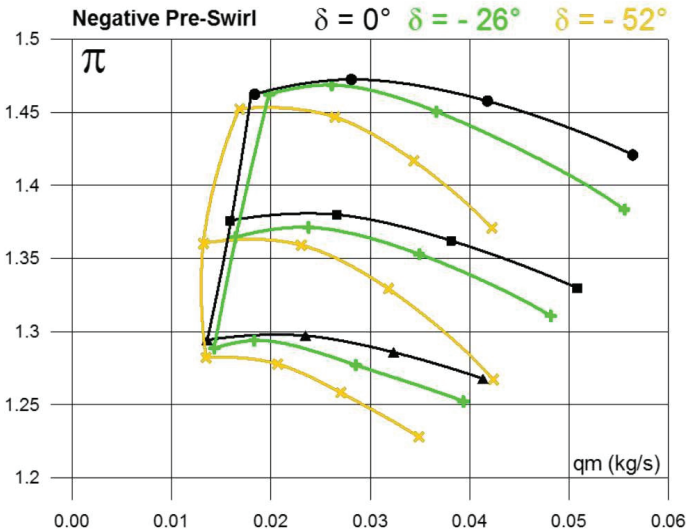

Fig. 4. Effect of different angles and direction of the pre-swirl on characteristic curves: (a) for a positive pre-swirl, (b) for a negative pre-swirl.

\subsection{Effect of the pre-swirl on the intensity of instabilities in the surge area}

The pressure and flow rate instabilities may be compared thanks to surge loops by analyzing the area. Indeed, the area of surge loops is sensitive to the intensity or to the dissipated energy during the compressor surge. Fig. 5 shows the evolution of pressures at the inlet and outlet of the compressor when the compressor operates in its surge area. The dissipated energy is then quantifiable with the loop describing the operating point.

Previous measurements, corresponding to five positions of the pre-swirl and three rotation speeds of the compressor, have been carried out by the surge loops recording (Figures 6 and 7). It progressively shuts the vane at the outlet of the compressor until a deep surge is reached simultaneously acting on the valve before the turbine to maintain a constant speed. Pressure measurements permit to calculate instantaneous compression ratio and air flow.

This zone corresponds to unstable operating points for which flow rate and compression ratio oscillate with a periodic signal described by a stable frequency.

In the case of a positive pre-swirl we can conclude that for any rotation regime, surge loop area decreases for a moderate pre-swirl at $\delta=+26^{\circ}$ but seems to increase slightly for $\delta=+52^{\circ}$. On the other hand, in the case of a negative pre-swirl, the surge loop area increases for $\delta=-$ $26^{\circ}$ and falls rapidly for $\delta=-52^{\circ}$.

The measurement of the unsteady flow rate seems to be biased for some values of the pre-swirl angle. The location of the inclined blades of the pre-swirl between the flowmeter and the compressor disturbs the flow when it becomes reversed during the surge instabilities. The differential sensor associated to the flow rate measurement is not able to evaluate clearly the pressure variations due to the pressure reducing orifice.

\section{Conclusions}

The measurements obtained on our surge test bench show that the pre-swirl does not only affect the appearance of the surge phenomenon, as it was shown in the literature for other different systems, but also on the intensity of the instabilities in the deep surge range. This information is a new important result that implies further studies of the optimal incidence angle of pre-swirl blades are needed. In order to improve our study, new experiments are in progress. In order to analyze more precisely the flow upstream blades of the compressor wheel, a pressure measurement can be insufficient. An optical access allowing PIV (Particle Image Velocimetry) or LDV (Laser Doppler Velocimetry) measurements should be considered to have more precise results which will be completed with CFD (Computational Fluid Dynamics) simulations.

Our first analysis shows that a positive pre-swirl is beneficial for pushing back the surge line limit and for increasing the compression ratio in the case of a low incidence angle of pre-swirl blades ( $+26^{\circ}$ for example). It also appears that for this angle, the adiabatic efficiency is slightly increased.

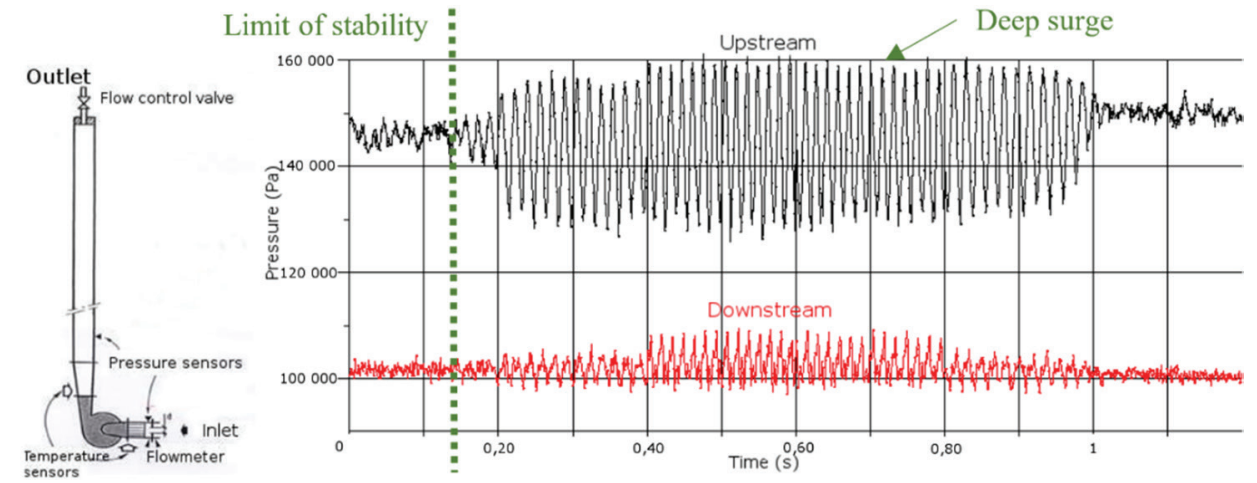

Fig. 5. Inlet and outlet pressure fluctuations during surge operation. 


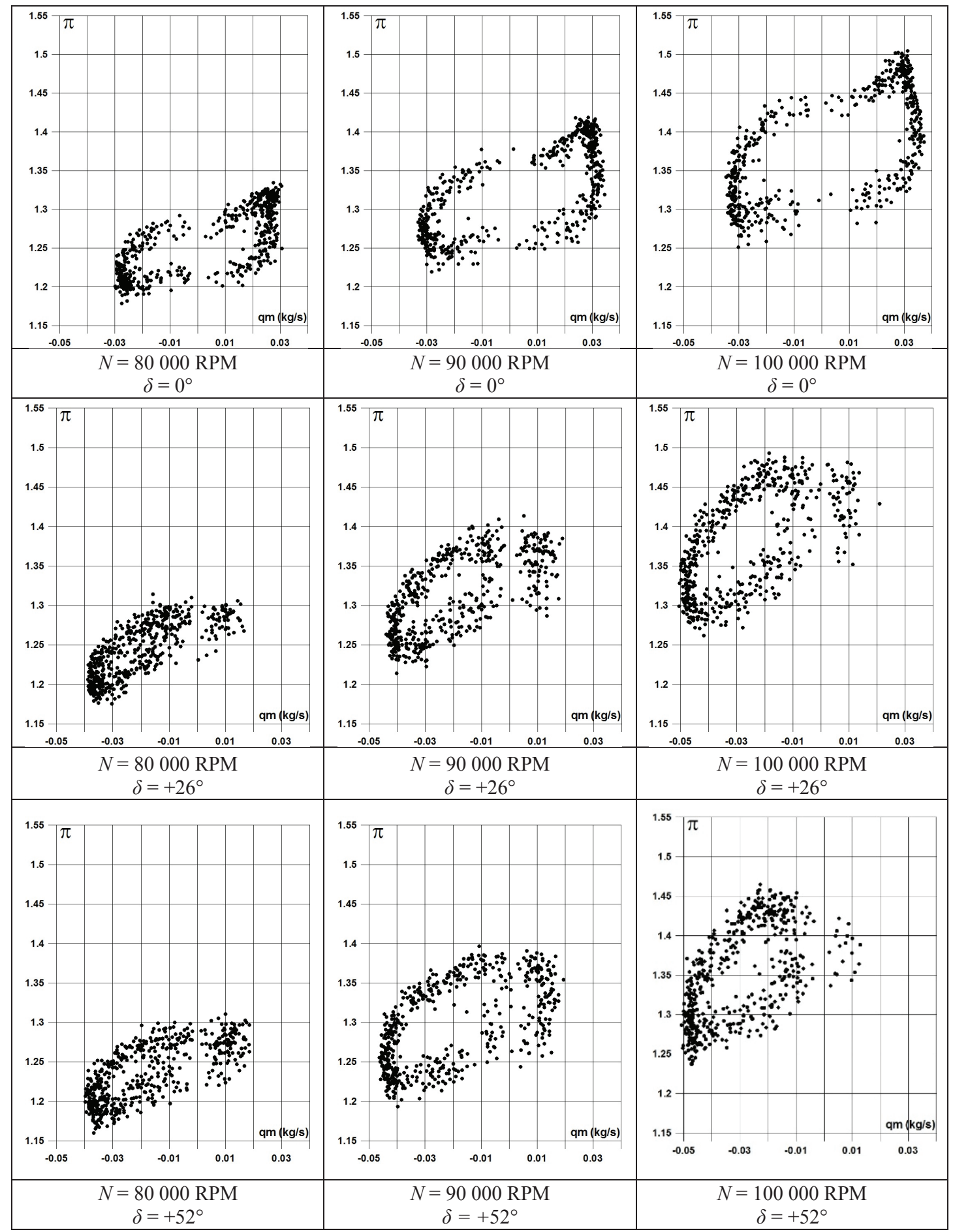

Fig. 6. Characteristic curves obtained in surge operation for a positive pre-swirl.

The authors would like to thank the Association Technique pour les Turbines A Gaz for its interest and for its logistic support.

\section{References}

1. R. Bidard, Compte-rendu de l'Académie des Sciences, Paris (1946)

2. J. Galindo, J. Serrano, X. Margot, A. Tiseira, N. Schorn, H. Kindl, Int. J. Heat Fuid Flow, 28 (2007)

3. J. Galindo, A. Tiseira, R. Navarro, D. Tari, C. Meano, App. Therm. Eng., 110 (2017)
4. J-M. Pugnet, Techniques de l'Ingénieur (2000)

5. M. Toussaint, M. Boudfar, CIEM2007, Bucarest, Romania (2007)

6. M. Toussaint, C. El Kamel, P. Aumoite, ISROMAC13, Honolulu, Hawaii (2010)

7. M. Toussaint, A. Lefebvre, G. Descombes, A. Marchal, COFRET12, Sozopol, Bulgaria (2012)

8. W. Yuchuan, T. Lei, Z. Baoshan, C. Shuliang, W. Binbin, JMES, 229, 18 (2015) 


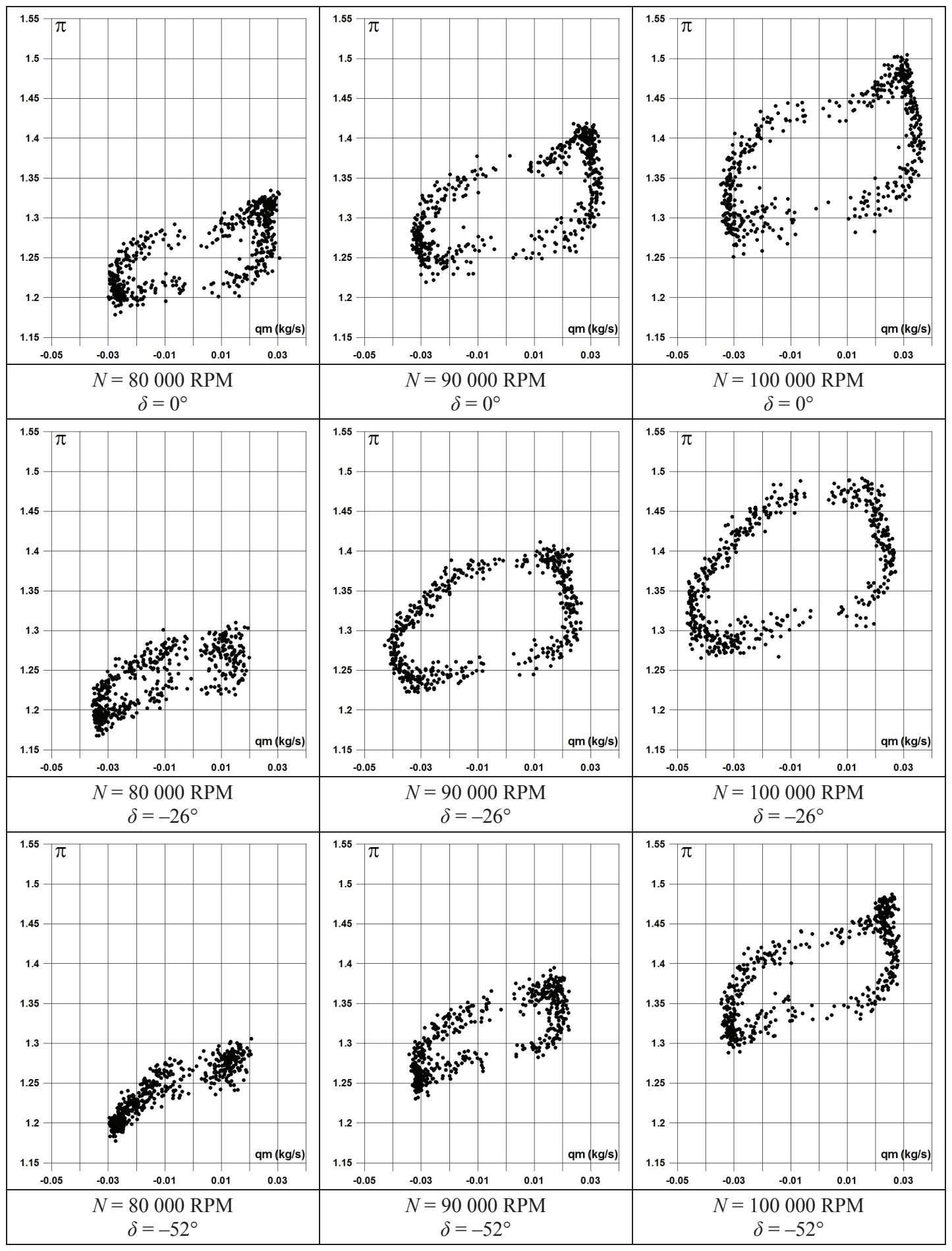

Fig. 7. Characteristic curves obtained in surge operation for a negative pre-swirl. 